\title{
Polymorphisms in the CD28/CTLA4/ICOS genes: role in malignant melanoma susceptibility and prognosis?
}

\author{
Marna G. Bouwhuis · Andreas Gast · Adina Figl • \\ Alexander M. M. Eggermont $\cdot$ Kari Hemminki · \\ Dirk Schadendorf $\cdot$ Rajiv Kumar
}

Received: 8 June 2009 / Accepted: 28 July 2009 / Published online: 12 August 2009

(C) The Author(s) 2009. This article is published with open access at Springerlink.com

\begin{abstract}
The appearance of vitiligo and spontaneous regression of the primary lesion in melanoma patients illustrate a relationship between tumor immunity and autoimmunity. T lymphocytes play a major role both in tumor immunity and autoimmunity. CD28, Cytotoxic T lymphocyte antigen 4 (CTLA4) and inducible costimulator (ICOS) molecules are important secondary signal molecules in the T lymphocyte activation. Single nucleotide polymorphisms (SNPs) in the CD28/CTLA4/ICOS gene region were reported to be associated with several autoimmune diseases including, type-1 diabetes, SLE, autoimmune thyroid diseases and celiac disease. In this study, we investigated the association
\end{abstract}

Electronic supplementary material The online version of this article (doi:10.1007/s00262-009-0751-2) contains supplementary material, which is available to authorized users.

M. G. Bouwhuis · A. M. M. Eggermont

Erasmus Medical Center-Daniel den Hoed Cancer Center,

Dr. Molewaterplein 50, 3015 GE Rotterdam, The Netherlands

A. Gast · A. Figl $~$ K. Hemminki $\cdot$ R. Kumar

Division of Molecular Genetic Epidemiology,

German Cancer Research Center (DKFZ),

Im Neuenheimer Feld 580, 69120 Heidelberg, Germany

D. Schadendorf

Department of Dermatology, University Hospital Essen,

Hufelandstraße 55, 45147 Essen, Germany

A. Gast

Department of Dermatology, University Hospital,

Theodor-Kutzer-Ufer 1-3, 68167 Mannheim, Germany

M. G. Bouwhuis ( $\square)$

Department of Surgery, Laboratory Experimental

Surgical Oncology, Section Surgical Oncology, Erasmus MC,

Dr. Molewaterplein 50, 3015 GE Rotterdam, The Netherlands

e-mail: m.bouwhuis@erasmusmc.nl of SNPs in the CD28, CTLA4 and ICOS genes with the risk of melanoma. We also assessed the prognostic effect of the different polymorphisms in melanoma patients. Twenty-four tagging SNPs across the three genes and four additional SNPs were genotyped in a cohort of 763 German melanoma patients and 734 healthy German controls. Influence on prognosis was determined in 587 melanoma cases belonging to stage I or II of the disease. In general, no differences in genotype or allele frequencies were detected between melanoma patients and controls. However, the variant alleles for two polymorphisms in the CD28 gene were differentially distributed in cases and controls. Similarly no association of any polymorphism with prognosis, except for the rs 3181098 polymorphism in the CD28 gene, was observed. In addition, individuals with AA genotype for rs11571323 polymorphism in the ICOS gene showed reduced overall survival. However, keeping in view the correction for multiple hypothesis testing our results suggest that the polymorphisms in the CD28, CTLA4 and ICOS genes at least do not modulate risk of melanoma and nor do those influence the disease prognosis in the investigated population.

Keywords Melanoma $\cdot$ Polymorphism $\cdot$ CD28/CTLA4/ ICOS $\cdot$ Susceptibility $\cdot$ Prognosis

\section{Introduction}

The association between tumor immunity and autoimmunity is complex [1]. Spontaneous regression is believed to be more common in melanoma than any other cancer types. However, the effect of the phenomenon on prognosis is rather unclear; however, the vitiligo is considered a favorable prognostic factor. Autoimmune conditions like thyroiditis and vitiligo, induced by interleukin 2 and/or 
Interferon $\alpha$ (IFN) therapy, have been associated with an improved prognosis in melanoma patients $[2,3]$. The appearance of autoantibodies or autoimmune manifestations in IFN-treated patients has been reported to be associated with significantly improved recurrence free and overall survival [4]. However, the findings could not be replicated when serum samples were analyzed of patients that were randomized to IFN treatment or observation in the EORTC 18952 and the Nordic Melanoma Group phase III trials [5].

$\mathrm{T}$ lymphocytes play an important role both in tumor immunity as well as in autoimmunity. The CD28, cytotoxic $\mathrm{T}$ lymphocyte antigen 4 (CTLA4) and inducible co-stimulator (ICOS) molecules are important secondary signaling molecules involved in the $\mathrm{T}$ lymphocyte activation. The genes encoding CD28, CTL4 and ICOS are located within a stretch of $300 \mathrm{~kb}$ region on chromosome 2q33. Ligation of CD28 molecules with the B7-1 (CD80) or B7-2 (CD86) on antigen presenting cells (APCs), stimulate $\mathrm{T}$ cell activation and proliferation. CTLA4 counterbalances this effect by competing with $\mathrm{CD} 28$ for B7-1/ B7-2 binding and is therefore an important inhibitor of $\mathrm{T}$ cell activation [6, 7]. CTLA-4 is also an established negative regulator of $\mathrm{T}$-cell function and proliferation through multiple mechanisms such as reducing interleukin (IL)-2 and IL-2 receptor productions and arresting T-cell at the G1-phase of cell cycle [8]. ICOS is another co-stimulatory molecule which is expressed on activated $\mathrm{T}$ cells. It binds to a unique ligand, ICOSL, and does not bind to other ligands such as B7-1/B7-2. Polymorphisms in the CD28/CTLA4/ICOS gene region have been associated with several autoimmune diseases including, type 1 diabetes, SLE, autoimmune thyroid diseases and celiac disease $[9,10]$. However, a majority of the studies focused on the known CTLA4 polymorphisms. A high prevalence of AA genotype for the CT60 polymorphism in the gene was observed in patients with renal cell cancer and a positive correlation between the polymorphism and tumor grade was also established [11]. The association between the variants in the promoter region of the CTLA4 gene and breast cancer progression has also been reported [12].

In this study, in order to find an association between polymorphisms in the CD28, CTLA-4 and ICOS genes and risk of cutaneous melanoma we screened patients from Germany and ethnically matched healthy controls. The single nucleotide polymorphisms (SNPs) in the three CD28/ CTLA4/ICOS genes were selected by tagging approach in order to cover the entire gene regions. Additionally four SNPs reported to be of interest in literature in the CTLA-4 gene were also included in the study. The association of variants alleles with prognostic outcome was also determined.

\section{Methods}

Patients and controls

The study population consisted of 763 melanoma patients from Germany (418 male and 345 female), recruited by the Skin Cancer Unit Mannheim, from 2001 to 2008. Patients with primary cutaneous melanoma with different disease stages that included, 10 cases with in situ melanoma, 615 with stage I/II, 111 stage III and 12 cases with stage IV of the disease. For 15 patients stage was unknown. Disease staging was performed according to the current AJCC criteria from 2001 [13]. Median and mean age of the melanoma cases at diagnosis was 55 and 54 years, respectively. Blood samples from case subjects were taken at their first presentation at the skin cancer unit. DNA was isolated from blood samples using Qiagen mini-preparation kits. Informed consent was obtained from the patients and the study was approved by the institutional ethical review board. Control subjects included 734 healthy German individuals (367 male and 367 female) recruited from blood bank Mannheim, with mean and median age of 60 and 61 years, respectively. They were born in southwest Germany and were matched for ethnicity with cases. The inclusion criteria for controls in the study included cancer free status. The age difference between the cases and controls was statistically significant ( $T$-test; $P$-value $<0.01)$, whereas, the gender difference was not statistically significant $\left(\chi^{2}\right.$-test; $P$-value $>0.05$ ).

Genes and SNPs selection

The selection of polymorphisms in the CD28, CTLA4 and ICOS genes was based on inclusion of known non-synonymous SNPs and those located in regulatory regions as reported in the dbSNP database of the National Center for Biotechnology Information, NCBI (http://www.ncbi.nlm. nih.gov/SNP/) or reported in published papers. Additionally, tagging SNPs from each gene region were selected from HapMap data using Haploview software 3.32, with pair-wise $r^{2}>0.8$ for each SNP pair and minor allele frequencies $>5 \%$ (Fig. 1). Ten tagging SNPs in the CD28 gene, five in the CTLA4 gene and 10 in the ICOS gene were selected from HapMap database that covered three genes completely. Four polymorphisms, rs11571319 (CT61), rs11571302 (JO31), rs7665213 (JO30) and rs11571297 (JO27) in the CTLA4 gene, which have been described to correlate with autoimmune disease(s) were also selected. The investigated polymorphisms span a region of $31.0 \mathrm{~kb}$ for the $\mathrm{CD} 28$ gene region, $6.1 \mathrm{~kb}$ for the CTLA4 gene region and $24.7 \mathrm{~kb}$ for the ICOS gene region. In total, 29 polymorphisms in three genes (CD28, CTLA4 and ICOS) were identified. 


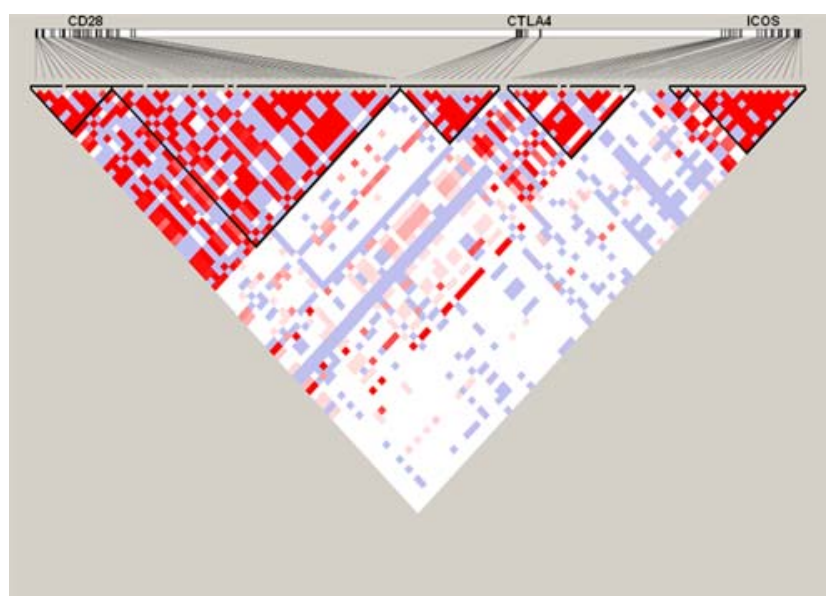

Fig. 1 Haplotype blocks in the genomic region with CTLA-4, CD28 and ICOS genes based on HapMap data

\section{Validation of the SNPs by DNA sequencing}

The validation of the 29 selected polymorphisms was carried out by sequencing a set of 32 DNA samples from control subjects. Sequencing reactions were performed using Big Dye Terminator Cycle Sequencing Kit (Applied Biosystems, Foster City, Ca, USA) and the following conditions were used; initial $94^{\circ} \mathrm{C}$ for 1 min followed by 27 cycles at $96^{\circ} \mathrm{C}$ for $16 \mathrm{~s}, 56^{\circ} \mathrm{C}$ for $5 \mathrm{~s}$ and $60^{\circ} \mathrm{C}$ for $4 \mathrm{~min}$. Reaction products were run on ABI prism 3100 Genetic analyzer (Applied Biosystems). Primers used in PCR amplification and sequencing reaction are listed in Supplementary Table.

\section{Genotyping}

Genotyping of the validated SNPs was performed by allelic discrimination technique (TaqMan assays, 'by demand or design' Applied Biosystems, Supplementary Table). Genotyping for one polymorphism in the ICOS gene (rs4355090) failed and was, thus, excluded from the study. Genotype failure rate was $0.14 \%$, calculated from samples that could not be genotyped after two repeated assays and by direct DNA sequencing. Genotyping data were confirmed by random direct DNA sequencing of $5 \%$ of all samples, which showed $100 \%$ concordance.

\section{Statistical analysis}

The association between malignant melanoma and different genotypes was estimated as odds ratios (OR), 95\% confidence intervals (CI) and $P$-values using SAS version 9.1. Estimates were adjusted for gender and age. Haplotype procedure of SAS/Genetics Software was used to calculate haplotype frequencies in cases and controls. Linkage disequilibrium (LD) was calculated with Haploview software (www.broad.mit.edu/mpg/haploview/documentation. php). The association between genotypes and different survival parameters, adjusted for age, gender and Breslow thickness, was carried out using proportional hazard regression (Cox) model. Metastases free survival (MFS) was the time from date of diagnosis until the first metastasis (either lymph node or distant metastasis) and overall survival (OS) was time from diagnosis to death. The follow-up of patients without metastases or who did not decease has been censored at the latest visit/last contact.

\section{Results}

Case-control study

The allelic distribution of polymorphisms in the CD28, CTLA4 and ICOS genes was assessed in 763 German melanoma patients and compared with 734 healthy German controls. A total of 28 SNPs were studied and genotype and allele distributions of all the polymorphisms are summarized in Table 1. Genotype frequency in controls for all the polymorphisms was in accordance with the Hardy-Weinberg equilibrium. Minor allele frequency (MAF) for the rs3181098 polymorphism was higher in cases than in controls (OR: $1.18,95 \%$ CI: $1.00-1.38 ; P=0.05$ ). And for the rs3181100 $(\mathrm{C}>\mathrm{G})$ polymorphisms the MAF was lower in cases than controls (OR: 0.83, 95\% CI: 0.71-0.97; $P=0.02$ ). None of the inferred haplotypes in three genes showed differential distribution between cases and controls (data not shown).

Association between polymorphisms and prognosis

The association between polymorphisms and survival parameters was evaluated for melanoma patients in stage I and II. Information regarding metastases free survival (MFS), overall survival (OS) and Breslow thickness was available for 587 patients ( 321 male and 266 female). Mean age was 54 years (median 55 years), the mean and median Breslow thickness was 1.84 and $1.50 \mathrm{~mm}$, respectively. Ulceration status of the primary tumor was not systematically recorded in the past and is therefore lacking. Age, gender and Breslow thickness were included as covariates in the Cox regression analysis. Overall, on comparing carriers versus non-carriers, no significant differences in OS were observed (Tables 2, 3, 4). A single SNP in the CD28 gene (rs3181098) showed an association with reduced metastases free survival (HR 1.34 95\% CI: 1.02-1.77). In addition to the carrier versus non-carrier approach, effect of the different genotypes on prognosis was analyzed. According to this analysis, one SNP (AA) in the ICOS gene 
Table 1 Case control

\begin{tabular}{|c|c|c|c|c|c|c|c|c|}
\hline SNP & Genotype & $\begin{array}{l}\text { Cases } \\
N=763\end{array}$ & $(\%)$ & $\begin{array}{l}\text { Controls } \\
N=734\end{array}$ & $(\%)$ & OR & $95 \% \mathrm{CI}$ & $P$-value \\
\hline \multicolumn{9}{|l|}{ CD28 } \\
\hline \multirow[t]{5}{*}{ rs3181098 } & GG & 315 & 41 & 331 & 45 & & & \\
\hline & $\mathrm{AG}$ & 331 & 43 & 325 & 44 & 1.05 & $0.84-1.31$ & \\
\hline & AA & 117 & 15 & 78 & 11 & 1.50 & $1.07-2.11$ & 0.06 \\
\hline & G-allele & 961 & 63 & 987 & 67 & & & \\
\hline & A-allele & 565 & 37 & 481 & 33 & 1.18 & $1.00-1.38$ & 0.05 \\
\hline \multirow[t]{5}{*}{ rs 3181100} & $\mathrm{CC}$ & 279 & 37 & 229 & 31 & & & \\
\hline & $\mathrm{CG}$ & 368 & 48 & 357 & 49 & 0.87 & $0.69-1.10$ & \\
\hline & GG & 116 & 15 & 145 & 20 & 0.68 & $0.50-0.93$ & 0.05 \\
\hline & C-allele & 926 & 61 & 815 & 56 & & & \\
\hline & G-allele & 600 & 39 & 647 & 44 & 0.83 & $0.71-0.97$ & 0.02 \\
\hline \multirow[t]{5}{*}{ rs3181101 } & $\mathrm{CC}$ & 570 & 75 & 559 & 77 & & & \\
\hline & $\mathrm{CG}$ & 175 & 23 & 165 & 23 & 0.98 & $0.76-1.26$ & \\
\hline & GG & 17 & 2 & 7 & 10 & 1.97 & $0.78-4.96$ & 0.35 \\
\hline & C-allele & 1,315 & 86 & 1,283 & 88 & & & \\
\hline & G-allele & 209 & 14 & 179 & 12 & 1.06 & $0.85-1.33$ & 0.61 \\
\hline \multirow[t]{5}{*}{ rs 1181390} & GG & 474 & 62 & 467 & 64 & & & \\
\hline & GT & 257 & 34 & 233 & 32 & 1.14 & $0.91-1.43$ & \\
\hline & $\mathrm{TT}$ & 32 & 4 & 33 & 5 & 0.92 & $0.55-1.55$ & 0.47 \\
\hline & G-allele & 1,205 & 79 & 1,167 & 80 & & & \\
\hline & T-allele & 321 & 21 & 299 & 20 & 1.06 & $0.88-1.28$ & 0.53 \\
\hline \multirow[t]{5}{*}{ rs 1181388} & GG & 575 & 76 & 545 & 74 & & & \\
\hline & $\mathrm{AG}$ & 169 & 22 & 170 & 23 & 0.98 & $0.76-1.26$ & \\
\hline & AA & 17 & 2 & 18 & 3 & 0.90 & $0.45-1.81$ & 0.95 \\
\hline & G-allele & 1,319 & 87 & 1,260 & 86 & & & \\
\hline & A-allele & 203 & 13 & 206 & 14 & 0.97 & $0.78-1.20$ & 0.76 \\
\hline \multirow[t]{5}{*}{ rs17533594 } & AA & 483 & 63 & 474 & 65 & & & \\
\hline & $\mathrm{AG}$ & 257 & 34 & 232 & 32 & 1.11 & $0.89-1.40$ & \\
\hline & GG & 23 & 3 & 24 & 3 & 0.94 & $0.52-1.73$ & 0.63 \\
\hline & A-allele & 1,223 & 80 & 1,180 & 81 & & & \\
\hline & G-allele & 303 & 20 & 280 & 19 & 1.06 & $0.88-1.28$ & 0.55 \\
\hline \multirow[t]{5}{*}{ rs3116494 } & AA & 414 & 54 & 393 & 54 & & & \\
\hline & $\mathrm{AG}$ & 307 & 40 & 299 & 41 & 1.01 & $0.81-1.26$ & \\
\hline & GG & 42 & 6 & 39 & 5 & 1.09 & $0.67-1.75$ & 0.94 \\
\hline & A-allele & 1,135 & 74 & 1,085 & 74 & & & \\
\hline & G-allele & 391 & 26 & 377 & 26 & 1.02 & $0.86-1.21$ & 0.79 \\
\hline \multirow[t]{5}{*}{ rs3181107 } & AA & 659 & 86 & 620 & 85 & & & \\
\hline & $\mathrm{AG}$ & 100 & 13 & 106 & 15 & 0.92 & $0.67-1.25$ & \\
\hline & GG & 4 & 1 & 6 & 1 & 0.74 & $0.20-2.79$ & 0.78 \\
\hline & A-allele & 1,418 & 93 & 1,346 & 92 & & & \\
\hline & G-allele & 108 & 7 & 118 & 8 & 0.91 & $0.68-1.20$ & 0.49 \\
\hline \multirow[t]{5}{*}{ rs3116496 (IVS3 + 17) } & $\mathrm{TT}$ & 487 & 64 & 475 & 65 & & & \\
\hline & $\mathrm{CT}$ & 254 & 33 & 231 & 32 & 1.10 & $0.88-1.38$ & \\
\hline & $\mathrm{CC}$ & 22 & 3 & 24 & 3 & 0.89 & $0.48-1.64$ & 0.63 \\
\hline & T-allele & 1,228 & 81 & 1,181 & 81 & & & \\
\hline & C-allele & 298 & 20 & 279 & 20 & 1.04 & $0.86-1.26$ & 0.66 \\
\hline
\end{tabular}


Table 1 continued

\begin{tabular}{|c|c|c|c|c|c|c|c|c|}
\hline SNP & Genotype & $\begin{array}{l}\text { Cases } \\
N=763\end{array}$ & $(\%)$ & $\begin{array}{l}\text { Controls } \\
N=734\end{array}$ & $(\%)$ & OR & $95 \% \mathrm{CI}$ & $P$-value \\
\hline \multicolumn{9}{|l|}{ CTLA4 } \\
\hline \multirow[t]{5}{*}{ rs 16840252} & $\mathrm{CC}$ & 521 & 68 & 489 & 67 & 1.00 & & \\
\hline & CT & 218 & 29 & 222 & 30 & 0.91 & $0.72-1.15$ & \\
\hline & $\mathrm{TT}$ & 23 & 3 & 21 & 3 & 1.02 & $0.54-1.91$ & 0.74 \\
\hline & C-allele & 1,260 & 83 & 1,200 & 82 & & & \\
\hline & T-allele & 264 & 17 & 264 & 18 & 0.95 & $0.78-1.15$ & 0.57 \\
\hline \multirow[t]{5}{*}{ rs5742909 (CT44) } & $\mathrm{CC}$ & 619 & 81 & 596 & 81 & 1.00 & & \\
\hline & CT & 136 & 18 & 130 & 18 & 0.96 & $0.73-1.27$ & \\
\hline & $\mathrm{TT}$ & 8 & 1 & 8 & 1 & 0.89 & $0.32-2.49$ & 0.95 \\
\hline & C-allele & 1,374 & 90 & 1,322 & 90 & & & \\
\hline & T-allele & 152 & 10 & 146 & 10 & 0.96 & $0.75-1.23$ & 0.74 \\
\hline \multirow[t]{5}{*}{ rs231775 (CT42) } & $\mathrm{AA}$ & 289 & 38 & 283 & 39 & 1.00 & & \\
\hline & $\mathrm{AG}$ & 369 & 48 & 345 & 47 & 1.08 & $0.86-1.36$ & \\
\hline & GG & 104 & 14 & 106 & 14 & 0.97 & $0.70-1.36$ & 0.71 \\
\hline & A-allele & 947 & 62 & 911 & 62 & & & \\
\hline & G-allele & 577 & 38 & 557 & 38 & 1.01 & $0.87-1.18$ & 0.90 \\
\hline \multirow[t]{5}{*}{ rs231777 } & $\mathrm{CC}$ & 539 & 71 & 514 & 70 & 1.00 & & \\
\hline & $\mathrm{CT}$ & 208 & 27 & 203 & 28 & 0.97 & $0.76-1.23$ & \\
\hline & $\mathrm{TT}$ & 15 & 2 & 16 & 2 & 0.83 & $0.39-1.77$ & 0.87 \\
\hline & C-allele & 1,286 & 84 & 1,231 & 84 & & & \\
\hline & T-allele & 238 & 16 & 235 & 16 & 0.95 & $0.78-1.17$ & 0.64 \\
\hline \multirow[t]{5}{*}{ rs3087243 (CT60) } & GG & 246 & 32 & 223 & 30 & 1.00 & & \\
\hline & $\mathrm{AG}$ & 355 & 47 & 388 & 53 & 0.81 & $0.63-1.03$ & \\
\hline & $\mathrm{AA}$ & 162 & 21 & 122 & 17 & 1.22 & $0.89-1.65$ & 0.01 \\
\hline & G-allele & 847 & 56 & 834 & 57 & & & \\
\hline & A-allele & 679 & 45 & 632 & 43 & 1.06 & $0.91-1.23$ & 0.45 \\
\hline \multirow[t]{5}{*}{ rs11571319 (CT61) } & GG & 518 & 68 & 488 & 67 & 1.00 & & \\
\hline & $\mathrm{AG}$ & 222 & 29 & 223 & 31 & 0.93 & $0.73-1.17$ & \\
\hline & $\mathrm{AA}$ & 23 & 3 & 21 & 3 & 1.02 & $0.54-1.92$ & 0.81 \\
\hline & G-allele & 1,258 & 82 & 1,199 & 82 & & & \\
\hline & A-allele & 268 & 18 & 265 & 18 & 0.96 & $0.79-1.16$ & 0.64 \\
\hline \multirow[t]{5}{*}{ rs11571302(JO31) } & GG & 225 & 30 & 210 & 29 & 1.00 & & \\
\hline & GT & 370 & 49 & 383 & 52 & 0.87 & $0.68-1.12$ & \\
\hline & TT & 168 & 22 & 140 & 19 & 1.14 & $0.84-1.55$ & 0.15 \\
\hline & G-allele & 820 & 54 & 803 & 55 & & & \\
\hline & T-allele & 706 & 46 & 663 & 45 & 1.05 & $0.90-1.22$ & 0.53 \\
\hline \multirow[t]{5}{*}{ rs7665213 (JO30) } & GG & 228 & 30 & 211 & 29 & 1.00 & & \\
\hline & AG & 370 & 49 & 383 & 52 & 0.87 & $0.68-1.12$ & \\
\hline & AA & 165 & 22 & 137 & 19 & 1.13 & $0.83-1.53$ & 0.17 \\
\hline & G-allele & 826 & 54 & 805 & 55 & & & \\
\hline & A-allele & 700 & 46 & 657 & 45 & 1.04 & $0.90-1.21$ & 0.59 \\
\hline \multirow[t]{5}{*}{ rs11571297 (JO27) } & $\mathrm{TT}$ & 214 & 28 & 193 & 26 & 1.00 & & \\
\hline & $\mathrm{CT}$ & 376 & 49 & 393 & 54 & 0.84 & $0.65-1.08$ & \\
\hline & $\mathrm{CC}$ & 173 & 23 & 148 & 20 & 1.07 & $0.79-1.45$ & 0.15 \\
\hline & T-allele & 804 & 53 & 779 & 53 & & & \\
\hline & C-allele & 722 & 47 & 689 & 47 & 1.02 & $0.88-1.18$ & 0.81 \\
\hline
\end{tabular}


Table 1 continued

\begin{tabular}{|c|c|c|c|c|c|c|c|c|}
\hline SNP & Genotype & $\begin{array}{l}\text { Cases } \\
N=763\end{array}$ & $(\%)$ & $\begin{array}{l}\text { Controls } \\
N=734\end{array}$ & $(\%)$ & OR & $95 \%$ CI & $P$-value \\
\hline \multicolumn{9}{|l|}{ ICOS } \\
\hline \multirow[t]{5}{*}{ rs10932029 (+173) } & $\mathrm{TT}$ & 538 & 71 & 488 & 67 & 1.00 & & \\
\hline & $\mathrm{CT}$ & 204 & 27 & 228 & 31 & 0.82 & $0.65-1.03$ & \\
\hline & $\mathrm{CC}$ & 21 & 3 & 15 & 2 & 1.14 & $0.57-2.27$ & 0.20 \\
\hline & T-allele & 1,280 & 84 & 1,204 & 82 & & & \\
\hline & C-allele & 246 & 16 & 258 & 18 & 0.89 & $0.73-1.08$ & 0.23 \\
\hline \multirow[t]{5}{*}{ rs4335928 } & $\mathrm{TT}$ & 579 & 76 & 559 & 76 & 1.00 & & \\
\hline & $\mathrm{CT}$ & 170 & 22 & 162 & 22 & 0.99 & $0.77-1.28$ & \\
\hline & $\mathrm{CC}$ & 14 & 2 & 12 & 2 & 1.11 & $0.50-2.47$ & 0.96 \\
\hline & T-allele & 1,328 & 87 & 1,280 & 87 & & & \\
\hline & C-allele & 198 & 13 & 186 & 13 & 1.01 & $0.80-1.26$ & 0.96 \\
\hline \multirow[t]{5}{*}{ rs 4675374} & $\mathrm{CC}$ & 457 & 60 & 436 & 60 & 1.00 & & \\
\hline & $\mathrm{CT}$ & 272 & 36 & 258 & 35 & 0.98 & $0.78-1.23$ & \\
\hline & $\mathrm{TT}$ & 34 & 5 & 38 & 5 & 0.85 & $0.52-1.40$ & 0.82 \\
\hline & C-allele & 1,186 & 78 & 1,130 & 77 & & & \\
\hline & T-allele & 340 & 22 & 334 & 23 & 0.96 & $0.80-1.14$ & 0.62 \\
\hline \multirow[t]{5}{*}{ rs7602383 } & AA & 550 & 72 & 533 & 73 & 1.00 & & \\
\hline & AG & 197 & 26 & 183 & 25 & 1.01 & $0.79-1.29$ & \\
\hline & GG & 16 & 2 & 17 & 2 & 0.93 & $0.46-1.90$ & 0.98 \\
\hline & A-allele & 1,297 & 85 & 1,249 & 85 & & & \\
\hline & G-allele & 229 & 15 & 217 & 15 & 0.99 & $0.81-1.23$ & 0.95 \\
\hline \multirow[t]{5}{*}{ rs 4521021} & $\mathrm{TT}$ & 450 & 59 & 451 & 61 & 1.00 & & \\
\hline & $\mathrm{CT}$ & 276 & 36 & 260 & 35 & 1.00 & $0.80-1.25$ & \\
\hline & $\mathrm{CC}$ & 37 & 5 & 23 & 3 & 1.47 & $0.83-2.60$ & 0.41 \\
\hline & T-allele & 1,176 & 77 & 1,162 & 80 & & & \\
\hline & C-allele & 350 & 23 & 306 & 21 & 1.07 & $0.89-1.28$ & 0.45 \\
\hline \multirow[t]{5}{*}{ rs 11571323} & GG & 587 & 77 & 534 & 73 & 1.00 & & \\
\hline & AG & 161 & 21 & 184 & 25 & 0.81 & $0.63-1.04$ & \\
\hline & AA & 15 & 2 & 13 & 2 & 1.30 & $0.60-2.84$ & 0.18 \\
\hline & G-allele & 1,335 & 88 & 1,252 & 86 & & & \\
\hline & A-allele & 191 & 13 & 210 & 14 & 0.89 & $0.71-1.10$ & 0.29 \\
\hline \multirow[t]{5}{*}{ rs 12466129} & $\mathrm{TT}$ & 448 & 59 & 451 & 62 & 1.00 & & \\
\hline & AT & 273 & 36 & 239 & 33 & 1.14 & $0.91-1.43$ & \\
\hline & AA & 42 & 6 & 42 & 6 & 0,94 & $0.59-1.51$ & 0.47 \\
\hline & T-allele & 1,169 & 77 & 1,141 & 78 & & & \\
\hline & A-allele & 357 & 23 & 323 & 22 & 1.06 & $0.89-1.27$ & 0.54 \\
\hline \multirow[t]{5}{*}{ rs10172036 } & GG & 301 & 40 & 283 & 39 & 1.00 & & \\
\hline & GT & 353 & 46 & 352 & 48 & 0.92 & $0.73-1.15$ & \\
\hline & $\mathrm{TT}$ & 109 & 14 & 97 & 13 & 1.04 & $0.75-1.45$ & 0.63 \\
\hline & G-allele & 955 & 63 & 918 & 63 & & & \\
\hline & T-allele & 571 & 37 & 546 & 37 & 0.99 & $0.85-1.16$ & 0.93 \\
\hline \multirow[t]{5}{*}{ rs10183087 } & AA & 461 & 61 & 418 & 57 & 1.00 & & \\
\hline & $\mathrm{AC}$ & 263 & 35 & 277 & 38 & 0.85 & $0.68-1.06$ & \\
\hline & $\mathrm{CC}$ & 38 & 5 & 37 & 5 & 1.04 & $0.63-1.70$ & 0.32 \\
\hline & A-allele & 1,185 & 78 & 1,113 & 76 & & & \\
\hline & C-allele & 339 & 22 & 351 & 24 & 0.92 & $0.77-1.10$ & 0.36 \\
\hline
\end{tabular}


Table 1 continued

\begin{tabular}{llrrrrrrr}
\hline SNP & Genotype & $\begin{array}{l}\text { Cases } \\
N=763\end{array}$ & $(\%)$ & $\begin{array}{c}\text { Controls } \\
N=734\end{array}$ & $(\%)$ & OR & $95 \%$ CI & $P$-value \\
\hline rs10932036 & AA & 611 & 80 & 594 & 81 & 1.00 & & \\
& AT & 144 & 19 & 129 & 18 & 1.09 & $0.83-1.44$ & \\
& TT & 7 & 1 & 7 & 1 & 1.12 & $0.37-3.35$ & 0.81 \\
& A-allele & 1,366 & 90 & 1,317 & 90 & & & \\
& T-allele & 158 & 10 & 143 & 10 & 1.08 & $0.85-1.39$ & 0.53 \\
\hline
\end{tabular}

Table 2 CD28

\begin{tabular}{|c|c|c|c|c|c|c|c|c|}
\hline \multirow[t]{2}{*}{ SNP } & \multirow[t]{2}{*}{ Genotype } & \multirow{2}{*}{$\begin{array}{l}\text { Cases }(\%) \\
N=587\end{array}$} & \multicolumn{3}{|c|}{ Metastases free survival } & \multicolumn{3}{|c|}{ Overall survival } \\
\hline & & & OR & $95 \% \mathrm{CI}$ & $P$-value & OR & $95 \% \mathrm{CI}$ & $P$-value \\
\hline \multirow[t]{2}{*}{ rs3181098 } & GG & $247(42)$ & & & & & & \\
\hline & AG/AA & $340(58)$ & 1.34 & $1.02-1.77$ & 0.04 & 1.18 & $0.81-1.72$ & 0.38 \\
\hline \multirow[t]{2}{*}{ rs3181100 } & $\mathrm{CC}$ & $212(36)$ & & & & & & \\
\hline & CG/GG & $375(64)$ & 0.82 & $0.62-1.08$ & 0.16 & 0.82 & $0.56-1.20$ & 0.31 \\
\hline \multirow[t]{2}{*}{ rs3181101 } & $\mathrm{CC}$ & $440(75)$ & & & & & & \\
\hline & CG/GG & $146(25)$ & 1.28 & $0.94-1.74$ & 0.12 & 1.21 & $0.80-1.83$ & 0.38 \\
\hline \multirow[t]{2}{*}{ rs1181390 } & GG & $360(61)$ & & & & & & \\
\hline & GT/TT & $227(39)$ & 0.92 & $0.70-1.21$ & 0.56 & 1.16 & $0.80-1.67$ & 0.45 \\
\hline \multirow[t]{2}{*}{ rs1181388 } & GG & $440(75)$ & & & & & & \\
\hline & AG/AA & $146(25)$ & 0.86 & $0.63-1.17$ & 0.32 & 0.82 & $0.53-1.25$ & 0.35 \\
\hline \multirow[t]{2}{*}{ rs17533594 } & $\mathrm{AA}$ & $372(63)$ & & & & & & \\
\hline & AG/GG & $215(37)$ & 1.03 & $0.78-1.35$ & 0.84 & 1.23 & $0.85-1.79$ & 0.28 \\
\hline \multirow[t]{2}{*}{ rs3116494 } & AA & $319(54)$ & & & & & & \\
\hline & AG/GG & $268(46)$ & 0.99 & $0.76-1.30$ & 0.96 & 1.13 & $0.79-1.62$ & 0.52 \\
\hline \multirow[t]{2}{*}{ rs3181107 } & AA & $506(86)$ & & & & & & \\
\hline & AG/GG & $81(14)$ & 0.81 & $0.54-1.21$ & 0.30 & 0.73 & $0.41-1.29$ & 0.28 \\
\hline \multirow[t]{2}{*}{ rs3116496 (IVS3 + 17) } & TT & $378(64)$ & & & & & & \\
\hline & $\mathrm{CT} / \mathrm{CC}$ & $209(36)$ & 1.16 & $0.88-1.53$ & 0.29 & 1.39 & $0.96-2.02$ & 0.08 \\
\hline
\end{tabular}

(rs11571323) was associated with reduced overall survival, $P=0.04$, HR 3.60; 95\% CI, 1.31-9.91, however, only 13 (2.2\%) melanoma patients carried AA genotype.

\section{Discussion}

The immunogenic nature of malignant melanoma is clinically manifested by spontaneous regression and appearance of vitiligo. The phenomenon of autoimmunity observed during various forms of immunotherapy, IL-2, IFN and anti-CTLA4 therapy, have been linked to the treatment response [2-4]. To understand the link between tumor immunity and autoimmunity in melanoma and to explore its implication on disease susceptibility and prognosis remains a challenge [14]. The results from studies evaluating polymorphisms in various autoimmune diseases suggest the existence of a common autoimmune disease locus in the CTLA4 gene [9].
We genotyped 28 polymorphisms located in the CD28, CTLA4 and ICOS genes in melanoma patients and healthy controls. Use of tagging approach covered the entire loci for all three genes. To the best of our knowledge, the screen for SNPS in the CTLA4 gene was the largest ever performed in melanoma patients (and controls) and the first one for the ICOS and CD28 genes. Our results showed that the variant alleles for two polymorphisms in the CD28 gene (rs3181098 and rs3181100) were differentially distributed in cases and controls. No differences in genotype or allele frequencies were detected between melanoma patients and controls for any other polymorphism. Similarly, carriers of the variant allele for the polymorphism rs3181098 in the CD28 gene showed reduced metastasis free survival and for the polymorphism rs11571323 the individuals with variant allele homozygous genotype were associated with reduced overall survival. However, keeping in view the number of tests carried out in the present study, the observed significant 
Table 3 CTLA4

\begin{tabular}{|c|c|c|c|c|c|c|c|c|}
\hline \multirow[t]{2}{*}{ SNP } & \multirow[t]{2}{*}{ Genotype } & \multirow{2}{*}{$\begin{array}{l}\text { Cases }(\%) \\
N=587\end{array}$} & \multicolumn{3}{|c|}{ Metastases free survival } & \multicolumn{3}{|c|}{ Overall survival } \\
\hline & & & OR & $95 \% \mathrm{CI}$ & $P$-value & OR & $95 \% \mathrm{CI}$ & $P$-value \\
\hline \multirow[t]{2}{*}{ rs 16840252} & $\mathrm{CC}$ & $399(68)$ & & & & & & \\
\hline & $\mathrm{CT} / \mathrm{TT}$ & $187(32)$ & 1.11 & $0.84-1.48$ & 0.46 & 1.25 & $0.85-1.82$ & 0.26 \\
\hline \multirow[t]{2}{*}{ rs5742909 (CT44) } & $\mathrm{CC}$ & $476(81)$ & & & & & & \\
\hline & $\mathrm{CT} / \mathrm{TT}$ & $111(19)$ & 1.24 & $0.88-1.72$ & 0.22 & 1.40 & $0.90-2.18$ & 0.14 \\
\hline \multirow[t]{2}{*}{ rs231775 (CT42) } & AA & $219(37)$ & & & & & & \\
\hline & $\mathrm{AG} / \mathrm{GG}$ & $367(63)$ & 0.99 & $0.75-1.30$ & 0.93 & 0.92 & $0.63-1.33$ & 0.64 \\
\hline \multirow[t]{2}{*}{ rs231777 } & $\mathrm{CC}$ & $414(71)$ & & & & & & \\
\hline & $\mathrm{CT} / \mathrm{TT}$ & $172(29)$ & 1.04 & $0.78-1.39$ & 0.79 & 1.23 & $0.83-1.80$ & 0.30 \\
\hline \multirow[t]{2}{*}{ rs3087243 (CT60) } & GG & $195(33)$ & & & & & & \\
\hline & AG/AA & $392(67)$ & 0.99 & $0.74-1.31$ & 0.92 & 0.93 & $0.63-1.36$ & 0.69 \\
\hline \multirow[t]{2}{*}{ rs11571319 (CT61) } & GG & $397(68)$ & & & & & & \\
\hline & AG/AA & $190(32)$ & 1.10 & $0.83-1.45$ & 0.51 & 1.19 & $0.81-1.73$ & 0.38 \\
\hline \multirow[t]{2}{*}{ rs11571302 (JO31) } & GG & $173(30)$ & & & & & & \\
\hline & GT/TT & $414(71)$ & 0.95 & $0.71-1.27$ & 0.72 & 0.80 & $0.55-1.17$ & 0.26 \\
\hline \multirow[t]{2}{*}{ rs7665213 (JO30) } & GG & $176(30)$ & & & & & & \\
\hline & AG/AA & $411(70)$ & 0.95 & $0.72-1.27$ & 0.74 & 0.83 & $0.57-1.21$ & 0.32 \\
\hline \multirow[t]{2}{*}{ rs11571297 (JO27) } & TT & $163(28)$ & & & & & & \\
\hline & $\mathrm{CT} / \mathrm{CC}$ & $424(72)$ & 0.87 & $0.65-1.16$ & 0.33 & 0.76 & $0.52-1.11$ & 0.16 \\
\hline
\end{tabular}

Table 4 ICOS

\begin{tabular}{|c|c|c|c|c|c|c|c|c|}
\hline \multirow[t]{2}{*}{ SNP } & \multirow[t]{2}{*}{ Genotype } & \multirow{2}{*}{$\begin{array}{l}\text { Cases }(\%) \\
N=587\end{array}$} & \multicolumn{3}{|c|}{ Metastases free survival } & \multicolumn{3}{|c|}{ Overall survival } \\
\hline & & & OR & $95 \% \mathrm{CI}$ & $P$-value & OR & $95 \% \mathrm{CI}$ & $P$-value \\
\hline \multirow[t]{2}{*}{ rs10932029 (+173) } & $\mathrm{TT}$ & $412(70)$ & & & & & & \\
\hline & $\mathrm{CT} / \mathrm{CC}$ & $175(30)$ & 0.87 & $0.65-1.17$ & 0.36 & 0.79 & $0.53-1.18$ & 0.25 \\
\hline \multirow[t]{2}{*}{ rs4335928 } & TT & $446(76)$ & & & & & & \\
\hline & $\mathrm{CT} / \mathrm{CC}$ & $141(24)$ & 1.17 & $0.86-1.60$ & 0.32 & 1.26 & $0.82-1.92$ & 0.29 \\
\hline \multirow[t]{2}{*}{ rs4675374 } & $\mathrm{CC}$ & $352(60)$ & & & & & & \\
\hline & $\mathrm{CT} / \mathrm{TT}$ & $235(40)$ & 1.08 & $0.82-1.42$ & 0.58 & 1.31 & $0.91-1.88$ & 0.15 \\
\hline \multirow[t]{2}{*}{ rs7602383 } & $\mathrm{AA}$ & $421(72)$ & & & & & & \\
\hline & $\mathrm{AG} / \mathrm{GG}$ & $166(28)$ & 1.02 & $0.76-1.37$ & 0.88 & 1.12 & $0.75-1.66$ & 0.59 \\
\hline \multirow[t]{2}{*}{ rs 4521021} & TT & $345(59)$ & & & & & & \\
\hline & $\mathrm{CT} / \mathrm{CC}$ & $242(41)$ & 0.96 & $0.73-1.26$ & 0.77 & 1.19 & $0.83-1.71$ & 0.35 \\
\hline \multirow[t]{2}{*}{ rs11571323 } & GG & $452(77)$ & & & & & & \\
\hline & AG/AA & $135(23)$ & 1.07 & $0.78-1.46$ & 0.70 & 1.27 & $0.83-1.94$ & 0.28 \\
\hline \multirow[t]{2}{*}{ rs12466129 } & $\mathrm{TT}$ & $340(58)$ & & & & & & \\
\hline & AT/AA & $247(42)$ & 0.86 & $0.66-1.13$ & 0.28 & 1.03 & $0.72-1.48$ & 0.88 \\
\hline \multirow[t]{2}{*}{ rs10172036 } & GG & $232(40)$ & & & & & & \\
\hline & GT/TT & $355(61)$ & 1.12 & $0.85-1.50$ & 0.42 & 0.86 & $0.58-1.26$ & 0.43 \\
\hline \multirow[t]{2}{*}{ rs10183087 } & $\mathrm{AA}$ & $352(60)$ & & & & & & \\
\hline & $\mathrm{AC} / \mathrm{CC}$ & $234(40)$ & 0.87 & $0.66-1.15$ & 0.33 & 0.93 & $0.64-1.35$ & 0.70 \\
\hline \multirow[t]{2}{*}{ rs 10932036} & AA & $467(80)$ & & & & & & \\
\hline & AT/TT & $119(20)$ & 0.76 & $0.54-1.06$ & 0.11 & 0.76 & $0.48-1.19$ & 0.23 \\
\hline
\end{tabular}

associations would be lost upon multiple hypothesis correction. Moreover, the detected association would also require confirmatory testing in an independent population.
One of the limitations of the present study included lack of pigmentation data, history of sunburns and the existence of statistical significant difference in mean age between 
cases and controls. Keeping in view the fact that ethnicity and not the age is major determinant of variant allele frequency, in our study design we ensured complete match between cases and controls for the latter parameter.

Our results are in accordance with a previous study that reported no difference in frequencies of six polymorphisms in the CTLA4 gene in 203 melanoma patients (stage IIb, IIc and III), compared to 288 healthy controls. Also no polymorphism correlated with improved recurrence free or overall survival [15]. However, several studies have reported association of the CTLA4 polymorphisms with other malignancies [16]. In humans cell CTLA4 exists in two isoforms, a full-length and a soluble isoform that lacks exon 3 due to alternative splicing [17]. The CT60 (A/G) polymorphism in the CTLA4 gene is a key susceptibility locus for autoimmune diseases, and the G-allele was shown to be correlated with decreased levels of the soluble isoform [9]. The frequency of the AA genotype for CT60 polymorphism was reported to be higher in renal cell carcinoma (RCC) patients than in controls. In addition, a positive correlation between the AA genotype and tumor grade was also observed, suggesting a role in tumor development [11]. The CT42 polymorphism (49A/G) in exon 1 is the only amino acid ( $\mathrm{Thr}>\mathrm{Ala}$ ) altering polymorphism in the CTLA4 gene; and the individuals homozygous for the Ala allele were associated with decreased CTLA4 expression on the T cell surfaces [18]. The AA genotype was correlated with increased frequencies in RCC patients and the A allele, in association with the $3^{\prime}$-untranslated region $(\mathrm{AT})_{82}$ alleles, correlated with non-Hodgkin's lymphoma (NHL) $[11,19]$. Interestingly, the GG variant was linked to an increased risk of gastric mucosa-associated lymphoid tissue (MALT) lymphoma [20]. In a study on multiple cancer types, 49A/G polymorphism has been risk of lung, breast and esophageal cancers as well as gastric cardia [16]. CTLA-4 with variant Thr allele has been shown to be associated with stronger inhibitory effect on T-cell activation than that with common allele. Polymorphisms in the promoter region of the CTLA4 gene were described to modulate expression of the gene [21]. This region contains the CT44 polymorphism $(-318 \mathrm{C} / \mathrm{T})$ variant. The $\mathrm{CC}$ genotype of the CT44 polymorphism was shown to be correlated with significantly reduced lymph node involvement in breast cancer patients [12]. The $\mathrm{T}$ allele was linked to an increased risk of B-CLL but to a decreased risk of MALT lymphoma [20, 22]. No correlation was found between the CT44 polymorphism and the risk of colon cancer [23]. The chromosomal region $2 \mathrm{q} 33$ containing the CTLA-4 and CD28 genes has been linked with asthma, however, the association with polymorphisms in the genes was not detected [24].

Melanoma patients with thick primary tumors and/or nodal involvement are at high risk for relapse or death [13].
However, adjuvant treatment is only beneficial in a small group of these patients. Genetic variability possibly predicts treatment outcome and could be a predictive marker to select the group benefiting from a certain treatment. In this study, only stage I and II melanoma patients were evaluated for a possible association between SNPs and prognosis. Since these patients do not frequently receive systemic treatment, we could not assess the predictive value of any of the polymorphisms. Nevertheless, recently it was shown that polymorphisms in the CTLA4 gene were correlated with response in melanoma patients (stage IV) receiving anti-CTLA4 treatment [25].

In conclusion, from the results of this large study we did not find convincing evidence for association between polymorphisms in the CD28, CTLA4 and ICOS genes and the risk of melanoma, nor with an effect on prognosis. Even two individual polymorphisms showed differential distribution of variant alleles between cases and controls, the effect nevertheless was marginal and a chance factor could no be ruled. The study was confined to German population, therefore, a strong association of polymorphisms investigated with melanoma susceptibility or disease outcome, in other populations cannot be entirely precluded.

Acknowledgments We acknowledge technical assistance by Sigrid Claus. This work was supported by the Initiative and Networking Fund of the Helmholtz Association within the Helmholtz Alliance on Immunotherapy of Cancer and an EORTC Translational Grant STrF/2008-1.

Open Access This article is distributed under the terms of the Creative Commons Attribution Noncommercial License which permits any noncommercial use, distribution, and reproduction in any medium, provided the original author(s) and source are credited.

\section{References}

1. Uchi H, Stan R, Turk MJ, Engelhorn ME, Rizzuto GA, Goldberg SM, Wolchok JD, Houghton AN (2006) Unraveling the complex relationship between cancer immunity and autoimmunity: lessons from melanoma and vitiligo. Adv Immunol 90:215-241

2. Atkins MB, Mier JW, Parkinson DR, Gould JA, Berkman EM, Kaplan MM (1988) Hypothyroidism after treatment with interleukin-2 and lymphokine-activated killer cells. N Engl J Med 318:1557-1563

3. Phan GQ, Attia P, Steinberg SM, White DE, Rosenberg SA (2001) Factors associated with response to high-dose interleukin-2 in patients with metastatic melanoma. J Clin Oncol 19:3477-3482

4. Gogas H, Ioannovich J, Dafni U, Stavropoulou-Giokas C, Frangia K, Tsoutsos D, Panagiotou P, Polyzos A, Papadopoulos O, Stratigos A et al (2006) Prognostic significance of autoimmunity during treatment of melanoma with interferon. N Engl J Med 354:709-718

5. Bouwhuis MG, Suciu S, Collette S, Aamdal S, Kruit WH, Bastholt L, Stierner U, Sales F, Patel P, Punt CJ et al (2009) Autoimmune antibodies and recurrence-free interval in melanoma patients treated with adjuvant interferon. J Natl Cancer Inst 101:869-877

6. Teft WA, Kirchhof MG, Madrenas J (2006) A molecular perspective of CTLA-4 function. Annu Rev Immunol 24:65-97 
7. Greenwald RJ, Freeman GJ, Sharpe AH (2005) The B7 family revisited. Annu Rev Immunol 23:515-548

8. Appleman LJ, Berezovskaya A, Grass I, Boussiotis VA (2000) CD28 costimulation mediates T cell expansion via IL-2-independent and IL-2-dependent regulation of cell cycle progression. J Immunol 164:144-151

9. Ueda H, Howson JM, Esposito L, Heward J, Snook H, Chamberlain G, Rainbow DB, Hunter KM, Smith AN, Di Genova G et al (2003) Association of the T-cell regulatory gene CTLA4 with susceptibility to autoimmune disease. Nature 423:506-511

10. Amundsen SS, Naluai AT, Ascher H, Ek J, Gudjonsdottir AH, Wahlstrom J, Lie BA, Sollid LM (2004) Genetic analysis of the CD28/CTLA4/ICOS (CELIAC3) region in coeliac disease. Tissue Antigens 64:593-599

11. Cozar JM, Romero JM, Aptsiauri N, Vazquez F, Vilchez JR, Tallada M, Garrido F, Ruiz-Cabello F (2007) High incidence of CTLA-4 AA (CT60) polymorphism in renal cell cancer. Hum Immunol 68:698-704

12. Erfani N, Razmkhah M, Talei AR, Pezeshki AM, Doroudchi M, Monabati A, Ghaderi A (2006) Cytotoxic T lymphocyte antigen-4 promoter variants in breast cancer. Cancer Genet Cytogenet 165:114-120

13. Balch CM, Buzaid AC, Soong SJ, Atkins MB, Cascinelli N, Coit DG, Fleming ID, Gershenwald JE, Houghton A Jr, Kirkwood JM et al (2001) Final version of the American Joint Committee on cancer staging system for cutaneous melanoma. J Clin Oncol 19:3635-3648

14. Ramirez-Montagut T, Turk MJ, Wolchok JD, Guevara-Patino JA, Houghton AN (2003) Immunity to melanoma: unraveling the relation of tumor immunity and autoimmunity. Oncogene 22:3180-3187

15. Gogas H, Metaxas Y, Dafni U, Polyzos A, Fountzilas G, Pectasides E, Skarlos D, Tsoutsos D, Panagiotou P, SpyropoulouVlachou M et al (2008) Evaluation of CTLA-4 polymorphisms in high-risk melanoma patients receiving adjuvant interferon. J Clin Oncol 26: Suppl; abstr 9054

16. Sun T, Zhou Y, Yang M, Hu Z, Tan W, Han X, Shi Y, Yao J, Guo Y, Yu D et al (2008) Functional genetic variations in cytotoxic T-lymphocyte antigen 4 and susceptibility to multiple types of cancer. Cancer Res 68:7025-7034
17. Magistrelli G, Jeannin P, Herbault N, Benoit De Coignac A, Gauchat JF, Bonnefoy JY, Delneste Y (1999) A soluble form of CTLA-4 generated by alternative splicing is expressed by nonstimulated human T cells. Eur J Immunol 29:3596-3602

18. Anjos S, Nguyen A, Ounissi-Benkalha H, Tessier MC, Polychronakos C (2002) A common autoimmunity predisposing signal peptide variant of the cytotoxic T-lymphocyte antigen 4 results in inefficient glycosylation of the susceptibility allele. J Biol Chem 277:46478-46486

19. Piras G, Monne M, Uras A, Palmas A, Murineddu M, Arru L, Bianchi A, Calvisi A, Curreli L, Gaviano E et al (2005) Genetic analysis of the 2q33 region containing CD28-CTLA4-ICOS genes: association with non-Hodgkin's lymphoma. Br J Haematol 129:784-790

20. Cheng TY, Lin JT, Chen LT, Shun CT, Wang HP, Lin MT, Wang TE, Cheng AL, Wu MS (2006) Association of T-cell regulatory gene polymorphisms with susceptibility to gastric mucosa-associated lymphoid tissue lymphoma. J Clin Oncol 24:3483-3489

21. Wang XB, Zhao X, Giscombe R, Lefvert AK (2002) A CTLA-4 gene polymorphism at position -318 in the promoter region affects the expression of protein. Genes Immun 3:233-234

22. Suwalska K, Pawlak E, Karabon L, Tomkiewicz A, Dobosz T, Urbaniak-Kujda D, Kuliczkowski K, Wolowiec D, Jedynak A, Frydecka I (2008) Association studies of CTLA-4, CD28, and ICOS gene polymorphisms with B-cell chronic lymphocytic leukemia in the Polish population. Hum Immunol 69:193-201

23. Dilmec F, Ozgonul A, Uzunkoy A, Akkafa F (2008) Investigation of CTLA-4 and CD28 gene polymorphisms in a group of Turkish patients with colorectal cancer. Int J Immunogenet 35:317-321

24. Heinzmann A, Plesnar C, Kuehr J, Forster J, Deichmann KA (2000) Common polymorphisms in the CTLA-4 and CD28 genes at $2 \mathrm{q} 33$ are not associated with asthma or atopy. Eur J Immunogenet 27:57-61

25. Breunis WB, Tarazona-Santos E, Chen R, Kiley M, Rosenberg SA, Chanock SJ (2008) Influence of cytotoxic T lymphocyte-associated antigen 4 (CTLA4) common polymorphisms on outcome in treatment of melanoma patients with CTLA-4 blockade. J Immunother 31:586-590 